\title{
Preface on special section on cognitive computing for emerging internet of things
}

\author{
Gustavo Ramirez-Gonzalez ${ }^{1}$ - Enas Adbulhay ${ }^{2}$
}

(c) Springer Science+Business Media, LLC, part of Springer Nature 2019

I am happy to introduce the special issue on Cognitive Computing for Emerging Internet of Things. We present a glimpse of the content of each of the paper that was accepted in this special issue.

Mazin Abed Mohammed et al. have taken one of the challenging issues in cancer diagnosis because of many conceivable shapes, regions, and image intensities. They have extracted features from cancerous NPC tumors, and then, a neural networkbased automated discrimination system was implemented for identification of malignant NPC tumors. The outcome of this work was evaluated using the efficiency, and the classifier has yielded more precision, sensitivity, and specificity compared to the other works in the literature.

Zhibin Zhang et al. have done research to enhance the robustness of fractal endpoint detection and make it applicable to more types of noise, fractal endpoint detection algorithm based on short-time domain. The multilabel algorithm represents signal energy distribution characteristics and strong periodicity and regularity of speech harmonic component with frequency domain and distinguishes speech from noise through extracting fractal dimension from short-time frequency domain energy distribution. The experimental results indicate short-time frequency domain fractal dimension method has better robustness.

Yifu Zeng et al. have performed research to improve the effectiveness of largescale flow data analysis. They have used a novel algorithm kdStreamSky algorithm to pre-calculate the state transition of the incremental data through event chain mechanism and accordingly update the state of the data point through event processing. Multiple pruning rules are proposed structurally for the clustering algorithm to narrow the search field and improve the search efficiency. A distributed hierarchical clustering method was adopted for the clustering analysis of individuals and the design of hierarchical forwarding scheme in this work.

Gustavo Ramirez-Gonzalez drgustavophd@gmail.com

1 Universidad del Cauca, Popayán, Colombia

2 Jordan University of Science and Technology, Irbid, Jordan 
Shankar et al. have taken his work on thyroid disease classification. The proposed model is to classify these thyroid data utilizing optimal feature selection and kernelbased classifier process. They have created classification models using "multikernel Support Vector Machine." The classification of the dataset is utilized to give better treatment, decision making and diagnose disease. The use of MKSVM classifier based on feature selection techniques for thyroid disease datasets attains optimal results with more accuracy compared to the other works in the literature.

Qianming Zhou et al. have improved the reliability and energy utilization efficiency mobile client data security storage protocol with multifactor node consideration on remote server. They have proposed the resampling mobile cloud data storage algorithm based on Gibbs probability allocation estimate. The Gibbs sampling process is used to realize high-dimensional coupling and non-supervised training for sample data in the process of allocation estimate. The experimental result shows that the proposed method has less energy consumption and higher storage reliability.

Li Ang et al. have studied the coding method of key frame and proposed a method of coding mode selection based on partition of frequency band. The low-frequency band and high-frequency band are encoded and decoded by Wyner-Ziv and intraframe, respectively. The decision of the coding mode is transferred to the decoder to improve the performance of the system without increasing the complexity of the encoder. In this proposed algorithm, the peak signal-to-noise ratio obtained by the proposed coding mode selection algorithm based on partition of frequency band is improved by $1-5 \mathrm{~dB}$.

Fanlei Yan has enhanced the effectiveness of UAV task planning optimization scheme. The optimization model was obtained through correlation function estimation and subsequent zero space projection. Then, Ant Colony Optimization (ACO) was used for solving multiobjective optimization problem so as to find out optimal character subset. Finally, the simulation experiment has verified the performance advantage of the proposed algorithm in the UAV task planning optimization scheme.

Bharathi et al. have done research in the area of cooperative cognitive radio networks. The multiobjective problems like energy consumption, queuing problems, priority levels of traffic classes, fairness, throughput, and user quality requirements have been addressed in this work. The load balance-enhanced particle swarm optimization algorithm for energy efficient cluster formation, which overcomes queuing problems, has been proposed, and multiple factors were considered for prioritizing the traffic rules. The results showed that the users achieve required resources via the proposed HCCRN, thus providing energy efficiency, fairness, throughput, and QoS.

Jing Yang et al. have improved the digital image's antipollution attack performance. They have proposed an encryption scheme with mixed homomorphic signature based on message authentication. The method creates a model for the coding process of the digital image by dint of source node set, non-source node set, and link set of directed multigraph. The work has guaranteed the integrity of the content in each MAC coding package and raises the execution efficiency of algorithm.

Renato F. Pereira et al. have done his paper on the microstructure of nodular cast irons. The disadvantage of reduction in tensile strength of the material as well as its yield limit has been overcome by computer vision algorithm to estimate the amount of degenerated graphite nodules. The proposed algorithm has been tested with two 
cast iron samples by measuring their microhardness and tensile strength. The results showed that the number of degenerated nodules is inversely proportional to the limit of traction resistance.

Prerna Sharma et al. have researched to predict the high resolution protein structure in the field of computational biology. As the properties of protein structure determine the quality and differentiate native structures from predicted structures, many classification models were presented in this field of research. They have proposed Improved Grey Wolf Optimization (IGWO) for the feature selection which ascertains optimal subset of features. Artificial Neural Network classifier predicts the protein structure with a maximum approximate accuracy compared to the previous works.

Jude D. Hemanth et al. have designed and developed an effective application for improving the diagnosis of heart diseases. They have dealt with the problem of heart sound analysis and disease diagnosis by using a mobile application that can perform the mentioned tasks by having also support from both virtual reality- and augmented reality-oriented components. It was also shown that employment of such system can be low cost according to chosen components.

Mengrul Wang has completed his research on Green building technology where the author has established an index system optimizing the green building design scheme. Author has determined the weights of various evaluation indexes and adopted a triangular classification building design example to establish the data flow control flow graph for data test and establish the data flow analysis of building design by aid of its dominator tree relation. It proves the effectiveness and practicality of the evaluation system based on live examples.

Sugumar et al. have experimented to provide a framework for providing optimal utilization of resources through a hypervisor technique. This frame work helps to reduce down time of resources and improve machine utilization. The frame work creates more than one virtual machine on a single physical machine. The performance of the partitioned machine is being examined using various signature cryptographic hash algorithms. Results show that the proposed framework is efficient and secure. The technique brings in reduced computation and supports better scalability.

Wang Tiancheng has experimented in his research work to improve non-supervision and monitoring effect for moving target of video, target tracking algorithm without supervision video based on resampling particle filter. The author has designed fast resampling particle filter algorithm based on quasi-Monte Carlo method for determining sequences for acquiring more uniform sample distribution in space. The experiment result shows that successful tracking to one or multiple moving targets can be realized for the method under general environment condition.

Joao Alexandre Lobo Marques et al. have presented new possibilities to improve computer-aided diagnostic systems. This work shows the nonlinear characterization of Fetal Heart Rate (FHR) and maternal UC signals as a first relevant step to consider the application of nonlinear metrics for medical decision support. The windowed entropy calculation is then presented following clinical interpretation of the FHR.

Yifu Zeng et al. have brought an solution to the problem of query optimization of database which requires high speed and high efficiency. The firefly group is divided 
into multiple groups with different parameters, and each group of fireflies followed the optimal firefly in the same group for optimizing. Then, mutual learning mechanism is established among various groups of optimal fireflies to realize inter-group information exchange. Experiment results indicate that MGFA is a query optimization method of database with good performance in this work.

Li Weizheng et al. have proposed an algorithm to optimize the network connectivity efficiency of a network with nodes of different energy harvesting rates by using the fewest RNs while ensuring a high success rate of data transmission. The Kruskal algorithm is used to create a minimum spanning tree (MST). The algorithm can be used to maintain network connectivity with the fewest RNs, which reduces the cost and increases the transmission success rate of data packages.

$\mathrm{Pu}$ Zaiyi has done his research on network intrusion which can be solved by dynamic Bayesian network and phase space reconstruction. The attacker's income, losses, costs, and encountered risks related to a cyber-attack are analyzed, an index system is established, and a dynamic Bayesian network is used to comprehensively assess the attack effects on network nodes to overcome drawbacks of the traditional node. The proposed method synthesizes more node information and observed data for the attack, thereby avoiding the discrepancy between actual attack effects and theoretical expectations of attacks from static assessment.

Publisher's Note Springer Nature remains neutral with regard to jurisdictional claims in published maps and institutional affiliations. 